\title{
Bronchogenic cyst of the neck occurring as a submandibular swelling in an adult: A case report based on a rare presentation
}

\author{
Ravisankar Nutalapati ${ }^{1}$, R.M.S.H.B. Medawela ${ }^{1, *}$, A. Manjula Attygalla ${ }^{1}$ and P.R Jayasooriya ${ }^{2}$
}

${ }^{1}$ Department of Oral Medicine and Periodontology, Faculty of Dental Sciences, University of Peradeniya, Peradeniya, Sri Lanka.

${ }^{2}$ Department of Oral Pathology, Faculty of Dental Sciences, University of Peradeniya, Peradeniya, Sri Lanka.

Received: 13/02/2019; Accepted: 10/05/2019

\begin{abstract}
Bronchogenic cysts are rare and benign congenital malformations. They arise from ventral surface of foregut during development and commonly present in mediastinum and intrapulmonary region. Bronchogenic cysts are often incidental findings in childhood. Majority of them are symptomless and rarely associated with symptoms such as stridor in neonates, dysphagia, dyspnea on exertion, and recurrent infection. Here we present a case of a bronchogenic cyst presented as Submandibular swelling in an adult a rare presentation.
\end{abstract}

Keywords: Bronchogenic Cyst, Submandibular Swelling, Congenital Malformation.

\section{INTRODUCTION}

Bronchogenic cysts are congenital malformations arising from tracheobronchial component during ventral foregut development.These malformations develop from diverticula or small buds (clusters of cells) that separate from the foregut in the embryogenesis of the tracheabronchial tree. They often communicate with trachea or bronchus and are of ectodermal and mesodermal origin (Ustundag et al., 2005). Bronchogenic cysts usually produce mild symptoms and intensity of the symptoms depend on the size and the location of the cyst. Majority are asymptomatic and predominantly localized in the mediastinum, and/or intrapulmonary region. Ultimate location of the cyst is determined by the timing of budding (St-Georges et al., 1991). Cervical bronchogenic cysts are predominantly reported in pediatric group and rarely in adult population (Kieran et al., 2010; Newkirk et al., 2004). They are often incidental findings in childhood. They may be associated with symptoms such as stridor in neonates, dysphagia, dyspnea on exertion, and recurrent infection (St-Georges et al., 1991). The purpose of this report is to describe an unusual presentation of asymptomatic cervical bronchogenic cyst as a submandibular swelling in an adult male. The Submandibular region is an uncommon location for the bronchogenic cyst and, it is a location that has not been reported in literature todate.

\section{Case presentation}

A 46-year-old Sri Lankan male presented to oral and maxillofacial Surgery unit, University of Peradeniya with a complaint of swelling on the right side submandibular region,which had been there for one month duration. History revealed a gradual onset of the swelling and patient was pain free throughout the course of the disease. He did not reveal a history of foul taste or meal time swelling. There were no signs of dysphagia or dyspnea.Patient was otherwise healthy and past medical and dental history did not reveal any significant contributions to the current presentation. The patient was a chronic smoker with no other risk habits such as non-smoking tobacco, areca nut or alcohol. Exploring his family history has not revealed similar pathologies affecting parents, siblings, and children.

A general examination of our patient revealed no significant findings and all vital signs (blood pressure, pulse rate, temperature, and respiratory rate) were within normal range. On inspection, a diffuse swelling was present lateral to the midline extending up to the angle of the mandible. Movements of the swelling observed with deglutition. Palpation revealed a firm, non pedunculated, 4 $\mathrm{X} 4 \mathrm{~cm}$ swelling. Further, smooth surfaced swelling showed regular margins. Bimanual palpation of submandibular gland has not elicited significant findings. No evidence of cervical lymphadenopathy was observed.

Routine hematological investigations were carried out (full blood count, serum electrolytes, and liver function test) with a renal function test and the results were well within limits.

Contrast Enhanced Computer Tomogram (CECT) revealed a soft tissue mass in the right thyroid region measuring $4.4 \times 4.1 \times 2.7 \mathrm{~cm}$. The lesion extended to the Submandibular region through thyrohyoid membrane and no evidence of bone erosion observed. There were no clear demarcation of planes between lump and submandibular gland. Overall CECT findings suggested a complicated cyst or a pharyngeal neoplasm.Further to arrive at a diagnosis fine needle aspiration cytology was performed and results were inconclusive.Therefore, the lesion was clinically 
suspected to be a throglossal duct cyst. Surgical excision was planned for total removal of the cyst under general anesthesia.

A Submandibular incision was placed and dissection extended up to the platysma. Platysma was separated. Subplatysmal dissection was done. On blunt dissection lesion was identified in the submandibular triangle deep to the digastric muscle. The lump was dissected deep up to the body of hyoid bone and removed (Figure 1 and 2). Layered suturing performed following satisfactory hemostasis at the surgical site. Recovery was uneventful. The excised lump was sent for histopathological diagnosis.
Formalin fixed specimen was dehydrated with alcohol and embedded in paraffin embedded. $4 \mu \mathrm{m}$ sections obtained with microtome and, $\mathrm{H}$ and $\mathrm{E}$ staining done following rehydration process.Histopathological sections showed a cyst with folded lining. Fibrous cyst wall consisted of fascicles of smooth muscle and it was lined by pseudo stratified columnar ciliated epithelium with goblet cells. Histopathological features were consistent with bronchogeniccyst (Figure $3 \mathrm{a}$ and $3 \mathrm{~b}$ ).

Patient had been routinely reviewed at the clinic with 1 month intervals, and he has been disease free for 6 months.

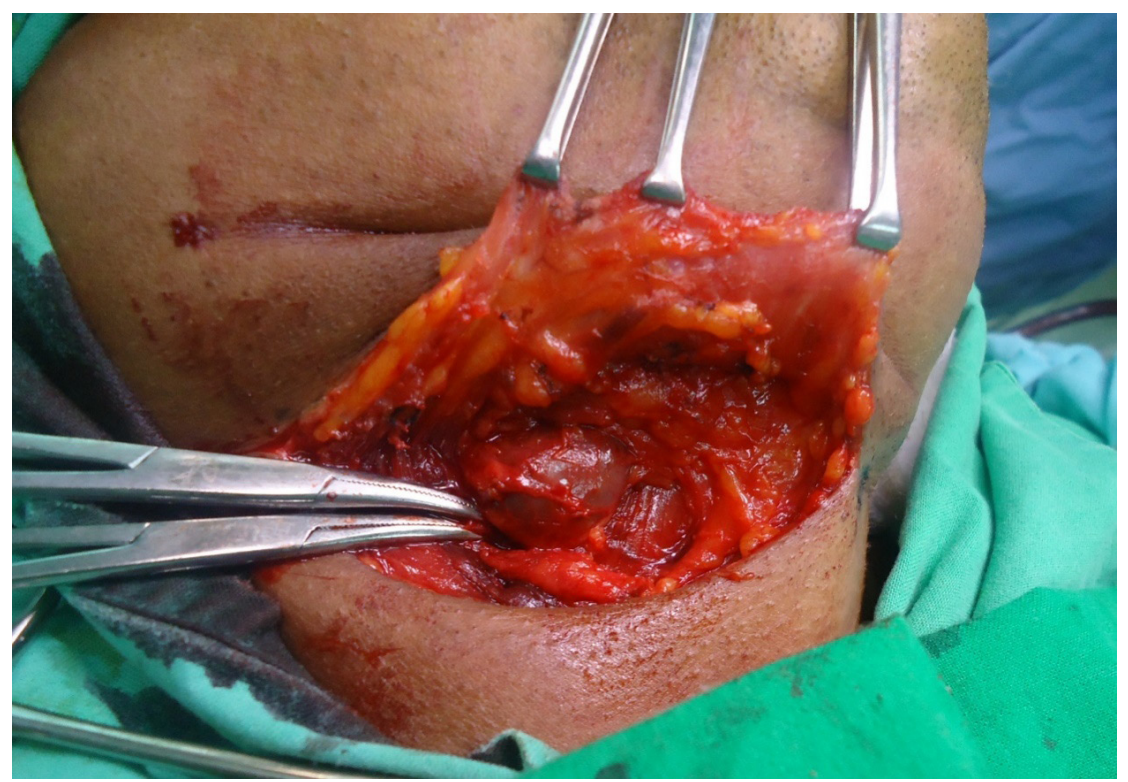

Figure 1: Symptomless swelling of gradual onset in submandibular region - Following sub-platysmal dissection to expose the lesion.

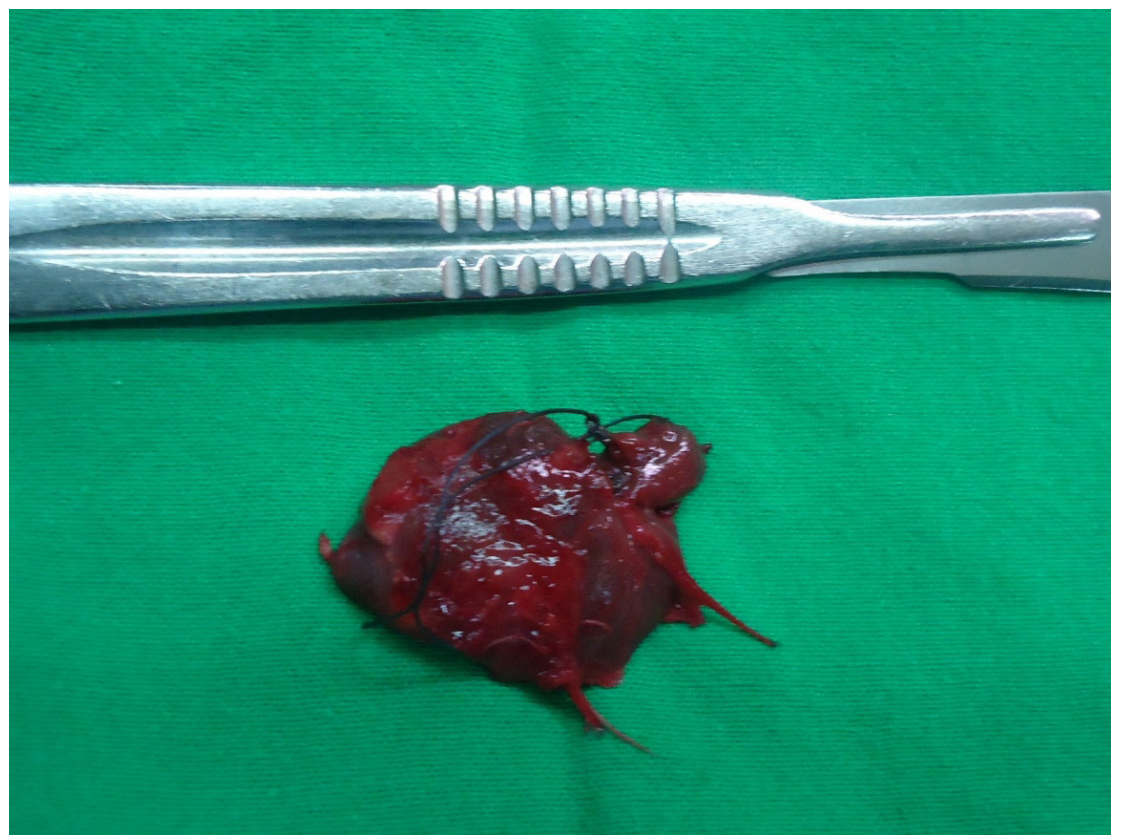

Figure 2: Lesion following surgical excision. 


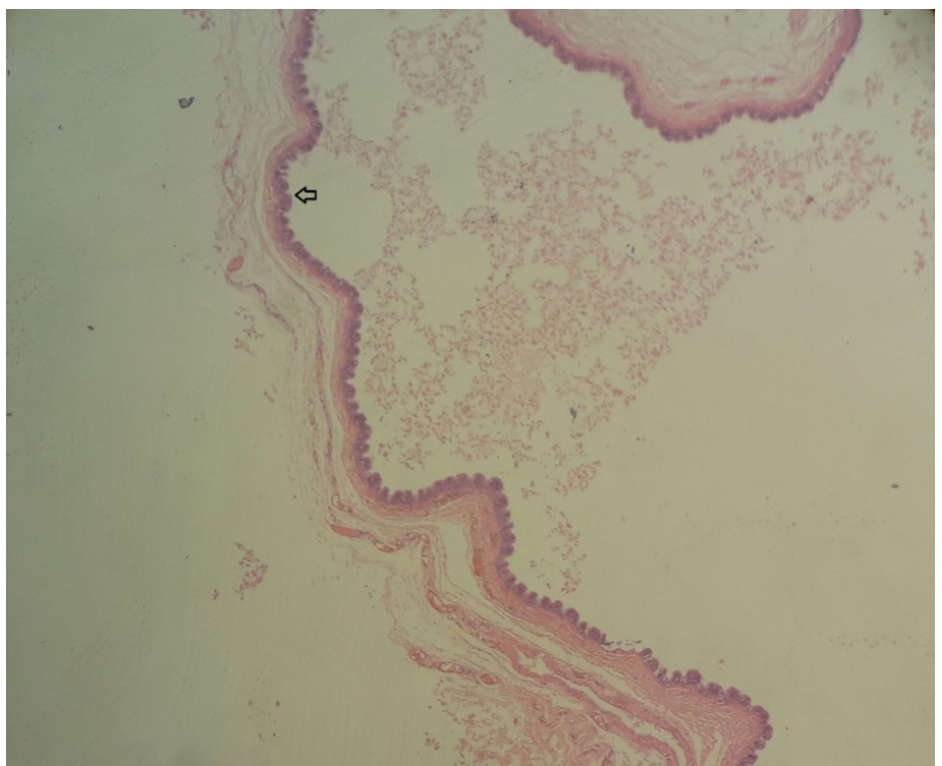

Figure 3(a): Histopathological section of Bronchogenic cyst: Psudostratifiedcolumnar ciliated epithelium lying on a fibro-muscular wall.

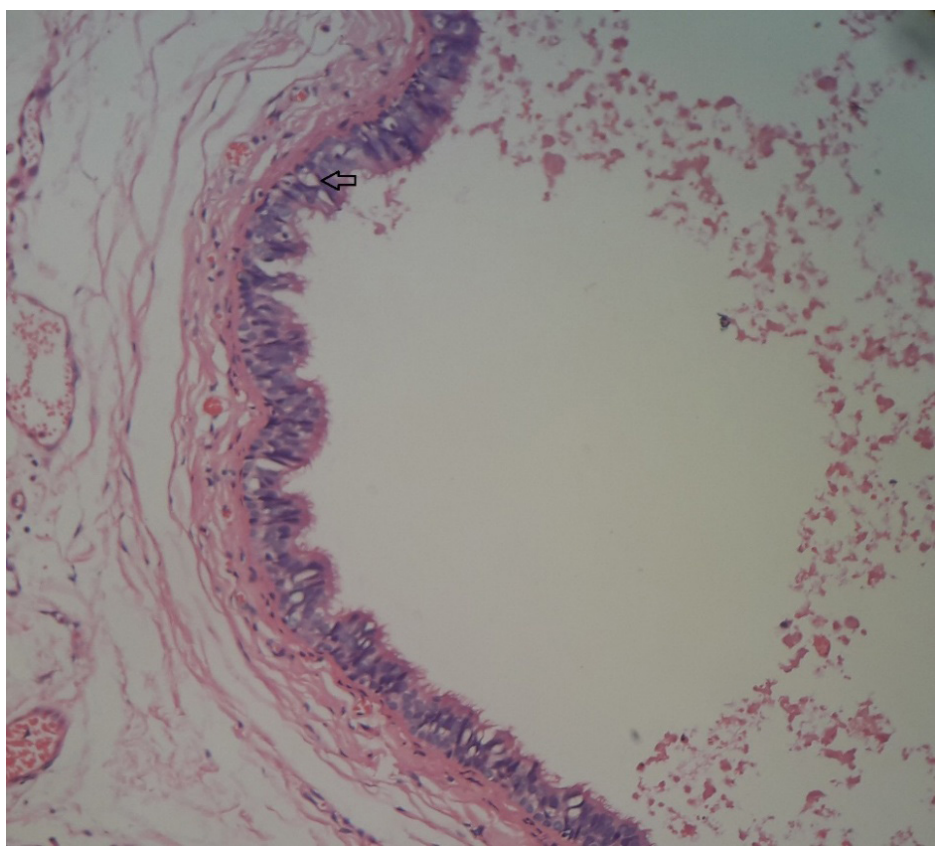

Figure 3 (b): Histopathological section of a cyst wall demonstrating goblet cells.

\section{DISCUSSION}

Bronchogenic cysts are rare benign lesions.In $5^{\text {th }}-7^{\text {th }}$ week during the embryogenesis of tracheobronchial tree, the primitive foregut is divided into dorsal esophagus and ventral trachea. Bronchogenic cysts are results of abnormal budding of primitive tracheobronchial tube at early stage of foetal development during the formation of ventral foregut (St-Georges et al., 1991; Newkirk et al., 2004; Akto_gu et al., 1996). Cysts formed due to heterotrophic rests of the foregut derived epithelium and these cysts can occur anywhere along the alimentary tract, the initial abnormal buds are located at higher levels and later ones are located peripherally (St-Georges et al., 1991). Based on origin of organs they are classified as Bronchogenic cysts, esophageal cysts and enteric duplication cysts (Kieran et al., 2010; Newkirk et al., 2004).
Bronchogenic cysts are commonly seen in childhood and rare in adults. When bronchogenic cysts emerge in adults, they commonly appear in the mediastinum and lung parenchyma with reported incidence rate of $1 / 42,000$ 68000 (Sanli et al., 2004). Ustundag et al., 2005 in his report on bronchogenic cysts included only 6 adult patients over the age of 25 years out of 45 patients. Moz et al., 2009 reviewed 14 cases of bronchogenic cysts of the neck in adults, of which 10 cases reported in midline, and 4 cases in lateral region most commonly in thyroid region and paratracheal region. Present case highlights a bronchogenic cyst located laterally and higher extending to submandibular triangle which the location is not reported to date to the best of our knowledge. Bronchogenic cysts of neck are usually asymptomatic, but if very large bronchogenic cysts produce symptoms like dyspnea, dysphagia, cough, 
respiratory distress and rarely secondary infection leading to abscess and fistula formation may occur (McManus et al., 1984).

Differential diagnosis of bronchogenic cysts include thymic cyst, branchial cleft cyst, thyroglossal duct cyst, papillary thyroid carcinoma-cystic variant, cystic hygromas, teratomas, cystic neuromas and tracheal diverticulum. Majority of above conditions present with cystic component and distinction is primarily based on histopathology (Newkirk et al., 2004). Clear description of the location and extent of the lesion, made by CECT directed the provisional diagnosis towards complicated cyst. However features revealedby the CECT such as extension into submandibular region from right thyroid region through thyrohyoid membrane was unusual. Therefore, histopathology is a gold standard for definitive diagnosis. Further, Literature suggests FNAC to be reliable diagnostic tool for neck lumps. However, aspiration histology was inconclusive in current case. This may be due to relatively few cells scattered in the cysts, as further elaborated in the literature (Akto_gu et al.,1996). Complete excision was attempted to prevent further recurrences and complication.

Histopathology of bronchogenic cysts shows a lining of pseudostratified ciliated columnar epithelium with cartilage, fibro muscular connective tissue and respiratory glands, which is suggestive of bronchial origin (Kieran et al, 2010; Newkirk et al., 2004). In present case the cyst is lined by pseudo stratifiedciliated columnar epithelium with goblet cells suggesting as bronchogenic cyst. In literature malignant transformation of bronchogenic cyst has been reported which is rare in cervical region and mostly seen in intra-thoracic cysts. The malignancies include squamous cell carcinoma, adenocarcinomas, mucoepidermoid carcinoma, sarcomas,anaplastic carcinoma etc. Tanaka et al, 1982 and Mizukami et al., 1984 reported a case of mucoepidermoid carcinoma arising from bronchogenic cyst. Tanita et al., 2002 presented a case of malignant melanoma arising from cutaneous bronchogenic cyst. Therefore, the possibility of malignant transformation emphasizes the importance of complete surgical excision of the cyst.

In conclusion, although bronchogenic cysts are rare in the neck particularly in submandibular region they should be included in the differential diagnosis of congenital malformation of upper lateral neck and submandibular region. Complete surgical excision is the treatment of choice based on risks of secondary infections, and malignant transformation.

\section{ACKNOWLEDGMENT}

Authors would like to acknowledge the guardians of patient and staff of Oral and maxillofacial surgery unit and Department of Oral pathology, Dental hospital, Peradeniya for their assistance in managing the patient and for comments that greatly improved the manuscript.

\section{REFERENCES}

Akto_gu, S., Yuncu, G., Halilc,olar H., Ermete, S. and Buduneli, T. (1996). Bronchogenic cysts: clinicopathological presentation and treatment. European Respiratory Journal., 9:2017-2021.

Calzada, A.P., Wu, W., Salvado, A.R., Lai, C.K. and Berke, G.S. (2011). Poorly differentiated adenocarcinoma arising from a cervical bronchial cyst. Laryngoscope, 121:1446-1448.

Kieran, S.M., Robson, C.D., Nose, V. and Rahbar, R., (2010). Foregut Duplication Cyst In Head And Neck:Presentation,Diagnosis And Management. Archives of Otolaryngology Head And Neck Surgery. 136(8):778-782.

Mc Manus K., Holt R., Aufdemorte, T.M. and Trinkle, J.K. (1984).Bronchogenic cyst presenting as deep neck abscess. Archives of Otolaryngology Head and Neck Surgery, 92:109-14.

Mizukami, Y., Matsubara, F. , Hashimoto, T. , Haratake, J., Terahata, S. , Noguchi, M. and Hirose, K. (1984), Primary mucoepidermoid carcinoma in the thyroid gland: A case report including an ultrastructural and biochemical study. Cancer, 53:1741-1745. doi:10.1002/1097-0142(19840415)53:8<1741::AIDCNCR2820530821>3.0.CO;2-C

Newkirk, K.A., Tassler, A.B., Krowiak, E.J. and Deeb, Z.E. (2004). Bronchogenic cysts of the neck inadults. Ann OtolRhinolLaryngol.; 113(9):691-5. Review. PubMed PMID:15453524.

Sanli, A., Onen, A., Ceylan, E., Yilmaz, E., Silistreli, E. and Açikel, U.(2004). A case of a bronchogenic cyst in a rare location. Annals of Thoracic Surgery, 77(3):1093e4.

St-Georges, R., Deslauriers, J., Duranceau, A., Vaillancourt, R., Deschamps, C., Beauchamp, G., Pagé, A. and Brisson, J. (1991). Clinical spectrum of bronchogenic cysts of themediastinum and lung in the adult. Ann Thorac Surg. Jul; 52(1):6-13. PubMedPMID: 2069465.

Tanaka, M., Shimokawa, R., Matsubara, O. et al. (1982) Mucoepidermoid carcinoma of the thymic region. ActaPatholJpn; 32:703-12.

Tanita, M., Kikuchi-Numagami, K., Ogoshi, K., Susuki, T., Tabata, N., Kudoh, K., et al. (2002). Malignant melanoma arising from cutaneousbronchogenic cyst of the scapular area. Journal of Americal academy of Dermatology, 46:19-21.

Moz, U., Gamba, P., Pignatelli, U., D’Addazio, G., Zorzi, F., Fiaccavento, S. and Milesi, F. (2009). Bronchogenic cysts of the neck: a rare localization and review of the literature. Acta Otorhinolaryngol Ital. Feb, 29(1):3640. Review. PubMed PMID: 19609381; PubMed Central PMCID: PMC2689566.

Ustundag, E., Iseri, M., Keskin, G., Yayla, B. and Muezzinoglu, B. (2005). Cervical Bronchogenic Cysts in Head And Neck Region. Journal of Laryngology and Otology, 119:419-423. 